\title{
Research on the Modularity Promotion of Green Building Life Cycle Cost Technology
}

\author{
Lin Feng ${ }^{1, a}$, Weiyi $\mathrm{He}^{1, \mathrm{~b}}$ \\ ${ }^{1}$ School of Tianjin University of Technology, Tianjin 300000, China; \\ a1358647619@qq.com, bweiyihe5@126.com
}

Keywords: LCC technology, Green building, Technology promotion, Percolation theory

\begin{abstract}
Analyze the popularization obstacles of LCC technology in the green building, put forward the LCC technology modular promotion mechanism based on the percolation theory. This paper builds the permeability model of LCC technology application, propose the generation rules of LCC module. Combined with the LCC technical process and the principle of module partition, decompose the LCC process to form the LCC module. Build the LCC technology expert system. The research aims to promote the popularization and application of LCC technology in the green building.
\end{abstract}

\section{Introduction}

Life Cycle Cost calculation as a kind of design scheme of economic evaluation system.[1]. In China, LCC technology import has lasted for more than 20 years, but so far, the trend of LCC adoption rate is slow. Green building show the concept of sustainable development, it is the inevitable trend to realized the transformation of the construction industry. But compared with the traditional architecture, green building have no advantage in investment cost, construction time and so on, therefore apply the LCC technology in the green building is very important to promote the development of green building. But at present, LCC technology application in the field of green building isn't ideal, so it is a realistic problem urgently to be solved. The study based on the percolation theory, according to the modular demand for the LCC technology promotion, aim to the LCC technology modular popularization mechanism, in order to provide the reference for LCC technology users.

\section{LCC technology popularization obstacles in green building under the perspective of technical innovation}

LCC is a process of system analysis, based on the purpose of looking for the best way to use the scarce resources, and evaluate all kinds of design or alternative plan. Basic processes of the LCC technology path include: (1)prepare the options(2)identify cost、cost breakdown structure (CBS); (3)build cost estimating model, form the predicted cost along the life-cycle cycle (Cost Modeling and Estimating /CME); (4)build the equivalent model to estimate cost in every life cycle time-point and calculate, form the comparable NPV or AV (LCC SUM); (5)sensitivity analysis; 6risk analysis; (7)build evaluation model、 evaluate、select, reach a optimized plan. In recent ten years, Christensen etc study the literature from 1977 to 2004 make the conclusion that step 1 to step 4 can take as the "Root Structure" of LCC, the other so-called method is the expansion of the "Root Structure" [2].

Because technology diffusion or new technology imitation constantly. Under the premise of scale, to form the promotion positive feedback mechanism of "innovation-imitate diffusion-re-innovation". Due to the economics connotation of "marginal cost" is the simple-copy costs of the increment. But construction project is complex, the user of the technology in order to 
guarantee the success of the LCC application, the application of LCC in any project would be the process of "innovation ---re-innovation", process of “imitated diffusion" is absent. In conclusion, the key to decide the success of LCC technology industrialization promotion is that imitable component is exist or not . It is also the key that if the LCC technology can be apply in construction project or not.

\section{LCC technology modular promotion path based on the percolation theory}

\subsection{LCC technology modular popularization mechanism}

"Percolation theory" is often used in engineering science, it is a natural model to describe many different phenomena[3]. In many ways the process of the percolation is similar with the transfer diffusion process, but in diffusion process, medium is passive, in the process of percolation, medium control the movement of the fluid.

According to the percolation theory, this paper take the LCC "Root Structure" as the medium of the technology system to analyze. The "root structure" is composed of CBS、CEM、LCC SUM. In different application situation and condition, method selection and matching is various. It cause that developed LCC technology path is inimitable, so that the technology application diffuse slowly. Thus, the LCC "root structure" is a kind of control retard medium, and take it as the mediating variable of the system. The variable can be the combination of any node on the LCC" root structure" network; but only in the certain good performance of combination circumstance, the corresponding technical path can be imitate by users, make the application state of the LCC transfer from the blocking state (0) into diffusion state (1), by this time the mediating variable is "percolation threshold" .

According to the above analysis, the general idea of the modularity promotion mechanism of LCC technology is: take the popularization obstacle factors of LCC as independent variable, take the any combination of the three link in the "root structure" as mediating variable, take the LCC application state as dependent variable, take the internal and external environment of LCC application as control variable, use the expert system to establish the relationship of independent variable--mediating variable--dependent variable, to decide the percolation threshold. In LCC "root structure", the node combination is called LCC module, thus building a LCC module system, finally forms the LCC technical promotion mechanism. The principle of how to form the LCC module:

1) Build the independent variable. On the basis of the previous research conclusion of this study[4]: feature of life-cycle cost structure weight distribution (X1)、data acquisition capability of the designer (X2)、computational capability of LCC (X3)、validity of analysis results (X4), the four obstacles determine different LCC technical path, as the independent variable. Establish the multilevel index set and scale for the above four independent variable respectively.

2 ) Build the dependent variable. designers evaluate the plan whether adopted the LCC successfully, namely the LCC application blocking(0), and diffusion state(1).

3 ) Build the mediating variable. Take the LCC "root structure” matrix (CBS-CME-LCCSUM), as the retard medium in the percolation relationship.

4) Build the multilevel index set about the LCC application performance , as the criterion of the percolation threshold.

5) Take the expert system as tool, take index and value of the four independent variable as the system input, expert system use the LCC application performance index in the repository. The system operate and submit various nodes combination plan of "root structure" . Through the interactive interface of the system and user, the plan designer choose these nodes combination plan, complete the LCC calculation and form LCC technical path in the situation of a certain independent 
variable, record by the expert system. The path in high repetitions which generate by the system, first that prove, in the percolation relationship, the dependent variable related with these certain combination is in diffusion state(1), therefore it has the property of percolation threshold; secondly, that prove these combination has good performance, reusable, namely the reusability is well, imitable, take these combination as LCC module.

In conclusion, LCC module is the part that can be reused. It is the key part that can reduce the LCC technology diffusion marginal cost and promote LCC technology industrialization promotion in the green building.

\subsection{Establish the LCC technology modular expert system}

This paper put forward the modular path, put the LCC technology into the traditional engineering economic consulting service, based on the reproducible LCC module, using the modular strategy to realize the mass customization of LCC application, and form the LCC technology application virtuous circle by bring the extra profit.

\subsubsection{Modularity of LCC calculation model selection}

Considering the green building situation, the customization dimensionality limit of use LCC technology professional engineering background, the LCC calculation results validity and data resources. According to the modularization design theory, the process of LCC calculation model selection break up into basic module 、 function module and structure module. The basic module to solve the situational problem of LCC technology, the function module and structure module through the interface rules to open the tight coupling of prediction technology and supporting data resources, make the system structure selection flexible、 the interface combination is simple.

\subsubsection{Modularity of LCC data resources}

Considering the supply model of all kinds of LCC calculation model supporting data resources, combined the three LCC technology requirement pattern in LCC technology market: 1)LCC service containing all data collection (turnkey contract model); 2) LCC service containing segmental data collection ( subcontracting model); 3 ) LCC service containing no data collection (parallel model). Respectively divided into three kinds of modules with data supporting function: turnkey service module、subcontracting service model and parallel service model. These three modules can guarantee the loose coupling of prediction technology and supporting data resources, thereby reduce the perceived risk of LCC technology potential users. Figure 1 is the concept map of the LCC technology based on modular design expert system integration pattern.

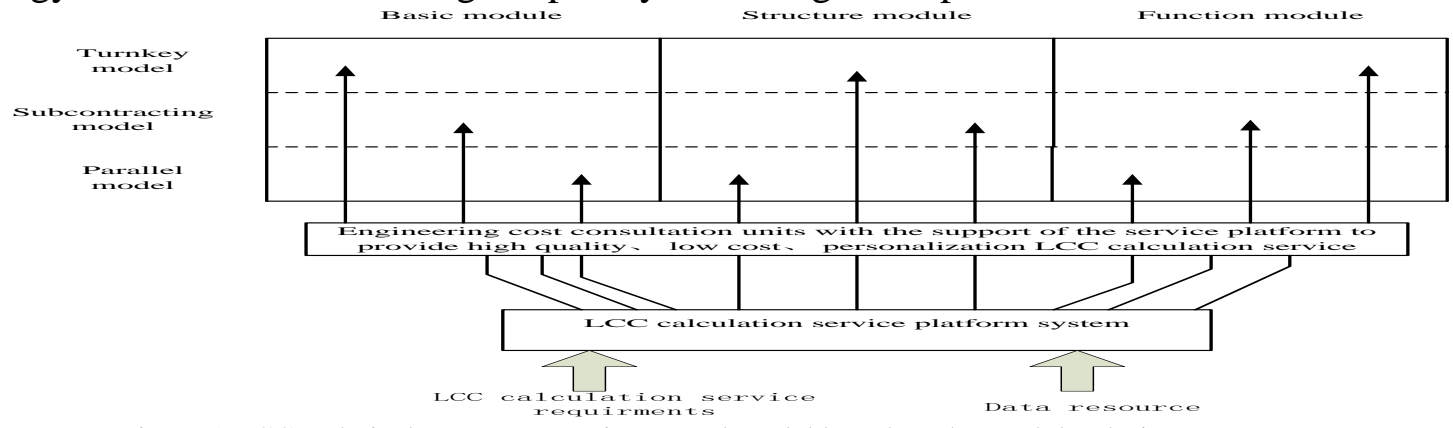

Figure 1 LCC technical expert system integrated model based on the modular design concept map

\section{Conclusion}

This study focus on problem solving LCC technology promotion in green building, put forward the LCC technology modular promotion model in the point and network green building situation. In the early stage of the project construction scheme evaluation decision stage, LCC modular technology promotion can promote the generation of life cycle cost saving oriented design product, can help to improve the control effect of project life-cycle cost. However, modular LCC technology popularization and application are still difficult, it also need to improve the LCC modular system 
application in the actual technical and economic evaluation of environmental effect, make it more adaptable to green building demand.

\section{Acknowledgement}

This study was supposed by 2015 Tianjin Philosophy and Social Sciences Planning Program (TJGL15-005)

\section{References}

[1] David G. Woodward. Life cycle costing-theory, information acquisition and application[J]. International Journal of Project Management, 1997, 15 (6) : 335-344.

[2] Christensen P N, Sparks G A, Kostuk K J. A method-based survey of life cycle costing literature pertinent to infrastructure design and renewal [J]. Journal of the Sanitary Engineering, 2005, 32: 250-259.

[3] Liu shengli, Feng huixia, Zhang jianqiang, The study and application of percolation theory[J]. Applied Chemical Industry, 2010, 39(7): 1074-1078.

[4] He Weiyi. To improve the performance of public projects path: promotion LCC mechanism research[D]. Tianjin: Tianjin University, 2010. 